\title{
PROJETO ESCOLAS NA UNIVERSIDADE ESTADUAL DE MARINGÁ: CRIAÇÃO DE UM AMBIENTE DE EXPERIMENTAÇÃO E EXPLORAÇÃO EM CIÊNCIAS
}

\author{
Gabriel da Cruz Dias \\ Universidade Estadual de Maringá \\ gabriel.fct@gmail.com \\ Cleiton Feitosa do Nascimento \\ Universidade Estadual de Maringá \\ feitocleiton@yahoo.com.br
}

\author{
José Cândido de Souza Filho \\ Universidade Estadual de Maringá \\ souza-jc@uol.com.br \\ Camila Muniz de Oliveira \\ Universidade Estadual de Maringá \\ camila_muniz98@outlook.com
}

Néryla Vayne Justino Alves Universidade Estadual de Maringá nerylaalves@yahoo.com.br

\section{Resumo}

A identificação de fenômenos através de experimentações e observações tem sido fato determinante ao longo da história para o desenvolvimento do conhecimento científico. Teorias são elaboradas por meio de experiências, e a compreensão delas fundamentam nossos caminhos e oferecem indicativos de nossa evolução. Porém, nos últimos anos tem se tornado um desafio despertar o interesse dos jovens pelo conteúdo científico que fundamentam a base do conhecimento em ciências. Neste projeto de extensão universitária, alunos graduandos do campus Regional da Universidade Estadual de Maringá (UEM) de Goioerê-PR confeccionaram, demonstraram e realizaram explicações prévias de experimentos de baixo custo juntamente com equipamentos do laboratório de física para alunos e professores do ensino médio e fundamental. Essas intervenções visam aproximar a universidade da comunidade escolar e criar um espaço onde os fenômenos antes trabalhados apenas em sala de aula são apresentados de forma diferente do cotidiano escolar.

Palavras-chave: Experimentação. Divulgação Científica. Ambientes Não Formais. Ciência. Física.

\section{DESIGN SCHOOLS IN MARINGÁ ESTADUAL UNIVERSITY: CREATION OF AN ENVIRONMENT OF EXPERIMENTATION AND EXPLORATION IN SCIENCES}

\begin{abstract}
The identification of phenomena by experimentation and observations has been determining factor throughout history for the development of scientific knowledge. Theories are elaborated by experiences and the understanding of them supports ours paths and offer indicative of our evolution. However, in recent years it has become a challenge to arouse young people's interest in the scientific content that underlies the basis of knowledge in science. In this project, undergraduate students from Maringá Estadual University (UEM) at Goioerê-PR prepare, demonstrate and carry out previous explanations of low-cost experiments in conjunction with physics laboratory experiments for students and teachers of the primary and high school. These interventions aim to bring the university closer to the school community and obtain a space where phenomena previously worked only in the classroom are presented differently from everyday school life.

Keywords: Experimentation. Scientific Divulgation. Not Formal. Science. Physics.
\end{abstract}

\section{PROYECTO ESCUELAS EN LA UNIVERSIDAD ESTADUAL DE MARINGA: CREACIÓN DE UN AMBIENTE DE EXPERIMENTACIÓN Y EXPLOTACIÓN EN CIENCIAS}

\section{Resumen}

La identificación de fenómenos a través de experimentos y observaciones ha sido un factor determinante a lo largo de la historia para el desarrollo del conocimiento científico. Teorías son elaboradas por medio de experiencias, y la comprensión de ellas fundamentan nuestros caminos y ofrecen indicativos de nuestra evolución. Sin embargo, en los últimos años se ha convertido en un desafío despertar el interés de los jóvenes por el contenido científico que fundamentan la base del conocimiento en ciencias. En este proyecto de extensión universitaria, alumnos graduados del campus Regional de la Universidad Estatal de Maringá (UEM) de Goioerê-PR confeccionaron, demostraron y realizaron explicaciones previas de experimentos de bajo costo junto con equipos del laboratorio de física para alumnos y profesores de la enseñanza media y fundamental. Estas intervenciones apuntan a acercar la universidad de la comunidad escolar y crear un espacio donde los fenómenos antes trabajados sólo en el aula se presentan de forma diferente al cotidiano escolar.

Palabras Clave: Experimentación. Divulgación Científica. Ambientes No Formales. La Ciencia. La Física. 
Projeto escolas na Universidade Estadual de Maringá: criação de um ambiente de experimentação e exploração em ciências

\section{INTRODUÇÃO}

Grande parte das escolas ainda adota o modelo positivista de ensino, em que há a transmissão do saber por parte do professor, e a recepção desse saber por parte do aluno. Nesse modelo, o aluno é análogo a uma máquina, a um sistema de entradas e saídas, cujas saídas podem ser controladas de fora (MOORE, 1982). O fluxo de informação ocorre em um sentido único e há a polarização vertical do conhecimento.

O aluno fica limitado a tarefas previamente elaboradas pelo professor, seguindo passo a passo esse "manual" que diz como as coisas devem ser feitas, a partir da reprodução de determinado fenômeno. Em direção oposta, fragilizando a concepção mecanicista, há a perspectiva orgânica, em que o foco é desviado para a necessidade de o aluno desenvolver sua maneira de trabalhar e adquirir conhecimento. (GASPAR, 2003).

No entanto, quando a questão se trata de investigar e redescobrir leis empíricas que apenas descrevem os fenômenos sem a formulação de hipótese explicativa, como, por exemplo, a formação de um arco-íris e o que faz uma bússola apontar sua agulha para uma determinada direção, este método é facilmente questionável, pois a formulação de uma lei científica, por exemplo, depende de conceitos científicos criados teoricamente, nos quais há implicitamente essa lei que expressa o fenômeno (GASPAR, 2003).

Nessa perspectiva, não se deixa (e não se deve) de lado a presença do professor, mas esse agora tem o seu papel modificado. Ele passa a ser um conselheiro, um consultor ou supervisor, atuando como um mediador entre o aluno e a tarefa, intervindo apenas nos momentos em que há indecisão ou falta de clareza. Seu objetivo deve ser deixar que o aluno ou o grupo assuma progressivamente o controle da atividade experimental (BORGES, 2003).

Por esse viés, a inserção do aluno em um ambiente sem a intenção de institucionalizá-lo, mas que o estimule a explorar, experimentar e a exercitar sua capacidade de raciocínio tornará sua educação muito menos uma transação (aluno-professor) do que um processo de descoberta da realidade (MOORE, 1982).

De acordo com Rodrigues (2009), a experimentação irá atuar, tanto no ensino de física como das ciências em geral como a mola-mestra, com a função de incentivar e despertar curiosidade do aluno. (RODRIGUES, 2009)

Para os alunos, o aprendizado de ciências ou mais especificamente da Física, é parte de um conjunto mais amplo de qualidades humanas, e contribui para melhorar a compreensão do mundo natural, desenvolvendo um sentido prático e analítico para a vida profissional. Porém, ao 
Projeto escolas na Universidade Estadual de Maringá: criação de um ambiente de experimentação e exploração em ciências

retomarmos um pouco de nossa história, vimos a inclusão da experimentação em sala de aula em poucas instituições de ensino (GASPAR, 2003).

Assim, a grande dificuldade está em estabelecer limites para essa orientação, já que esse procedimento pode ser caracterizado como um retrocesso às atividades dirigidas, contexto no qual a própria ideia de redescoberta foi proposta. Embora as atividades experimentais sejam uma necessidade incontestável, sua utilização em sala de aula ainda é inexpressiva em qualquer nível de ensino. (MENDONÇA \& DIAS, 2016).

Contudo, apesar do caráter interdisciplinar da Física, muitos professores atuantes não buscam uma correlação dessa ciência com as demais e também com as áreas tecnológicas. Isso contribui para não despertar interesse nos estudantes de nossa atual realidade. Esse, provavelmente, é um dos pontos que aumenta as dificuldades no ensino-aprendizagem de disciplinas científicas (em destaque a Física) nas séries fundamentais, secundárias e em nível de graduação. Enfatiza-se também que a falta de interesse pela Física nesses anos de vida escolar reflete diretamente no interesse, por parte de nossos jovens, em cursar licenciatura ou bacharelado nesta área.

Mesmo não havendo um consenso sobre as atividades que são responsáveis por promover a Física, existe consonância à sua necessidade, visando principalmente despertar o interesse e a curiosidade sobre os conteúdos científicos nas crianças e jovens, e principalmente, promover a popularização da ciência para a sociedade em geral, promovendo-a em ambientes que extrapolam o espaço escolar (MARANDINO et al, 2003).

Qualquer atividade contextualize e promova a compreensão de algum fenômeno, mesmo que ocorra fora do currículo padrão de uma instituição escolar, pode ser tratada como aprendizado informal (LIVINGSTONE, 1999). Evidentemente, situações corriqueiras e cotidianas problematizadas para um grupo de alunos pode ser uma oportunidade latente para o aperfeiçoamento do conhecimento científico. (SANTOS, PEREIRA \& PENIDO, 2011).

A aprendizagem informal baseia-se em oportunidades e processos naturais que surgem no cotidiano do indivíduo, o que lhe ocorreu durante sua vivência (FLACH \& ANTONELLO, 2010), enquanto a aprendizagem não formal extrapola os limites da sala de aula, o giz e a lousa, com marco para início e fim. A aprendizagem não formal baseia-se na cultura e prática dos indivíduos envolvidos, como por exemplos museus de história ou museus científicos, zoológicos, noites de observação celeste ou visitas em determinadas instituições ou circuitos científicos. (LANGHI, R. \& NARDI R. 2009). 
Projeto escolas na Universidade Estadual de Maringá: criação de um ambiente de experimentação e exploração em ciências

Desta maneira informal ou não formal, a aprendizagem irá ocorrer naturalmente e permitirá a busca do entendimento e habilidade que acontecem além dos programas de ensino formais já conhecidos (MARDEGAN, 2013).

O projeto intitulado Escolas na UEM-CRG (Universidade Estadual de Maringá - Campus Regional de Goioerê) justifica-se por buscar, por meio da experimentação no ensino de Física, não apenas a ilustração e facilitação do entendimento de algum fenômeno, mas incentivar alunos e professores a se desvencilharem do formalismo da estrutura escolar convencional de ensino que se tem praticado.

Neste projeto, desenvolvem-se atividades de divulgação científica propostas pelo grupo de extensão que compreende visitas a um laboratório didático com experimentos simples, mas que englobam diversas áreas da Física, observações astronômicas, cinema no campus e participações em feiras de ciências.

\section{O PROJETO NA PRÁTICA}

Com o objetivo de minimizar a problemática descrita no capítulo anterior, atividades típicas de caráter coletivo para divulgação de ciências têm sido incentivadas. Por exemplos, a criação de uma feira de ciências no âmbito escolar e realizações de noite de observação celeste com a utilização de telescópios (GOHN, 1999).

O aumento considerável de museus móveis e itinerantes é resultado da mobilização de várias universidades, instituições de pesquisa e secretarias estaduais, dentre outras, com o objetivo de irradiar acervos e exposições científicas, principalmente promover a divulgação científica para populações sem acesso, tanto geográfica como socialmente, a equipamentos científicos ou a materiais que exemplifiquem fenômenos científicos que os acercam. (PIERRO, 2015)

Em particular, no campus da UEM-CRG, organizam-se reuniões e encontros mensais para dar andamento ao projeto (objeto deste trabalho) que já tem duração de aproximadamente três anos. A equipe conta com dois bolsistas do programa institucional de bolsas de inclusão social da Fundação Araucária (PIBIS-FA-UEM), seis estudantes dos cursos de graduação do campus e seis professores orientadores. Nas reuniões, são planejadas demonstrações de Física, apresentações de teorias, criações de experimentos, seminários, minicursos, noites de observações e participações em eventos. 
Projeto escolas na Universidade Estadual de Maringá: criação de um ambiente de experimentação e exploração em ciências

Para as demonstrações de física, a apresentação de cada experimento envolve uma discussão prévia com os alunos a respeito do fenômeno que será observado, além de abordar uma possível situação no cotidiano e uma área de aplicação tecnológica.

Os graduandos confeccionam ou preparam, realizam as demonstrações e oferecem explicações prévias para professores e alunos do ensino fundamental e médio, com experimentos didáticos obtidos a partir de materiais de baixo custo e equipamentos do laboratório de Física. As visitas das escolas são agendadas frequentemente duas vezes ao mês.

No espaço criado no campus, assim como em um museu de ciências $^{1}$, existem diversos experimentos, aparatos envolvendo diversos campos da física, tais como óptica, mecânica eletricidade e ciências em geral (CEZALLI, 2002).

A Figura 1 apresenta uma bancada do laboratório didático com alguns experimentos que são utilizados para demonstrações, quando ocorrem visitas de estudantes do ensino médio ao campus, além da dinâmica do grupo.

Durante as visitas o atendimento ao público no campus é padronizado, possuindo duração de aproximadamente duas horas. Inicialmente realiza-se uma prévia para apresentação do projeto e dos monitores.

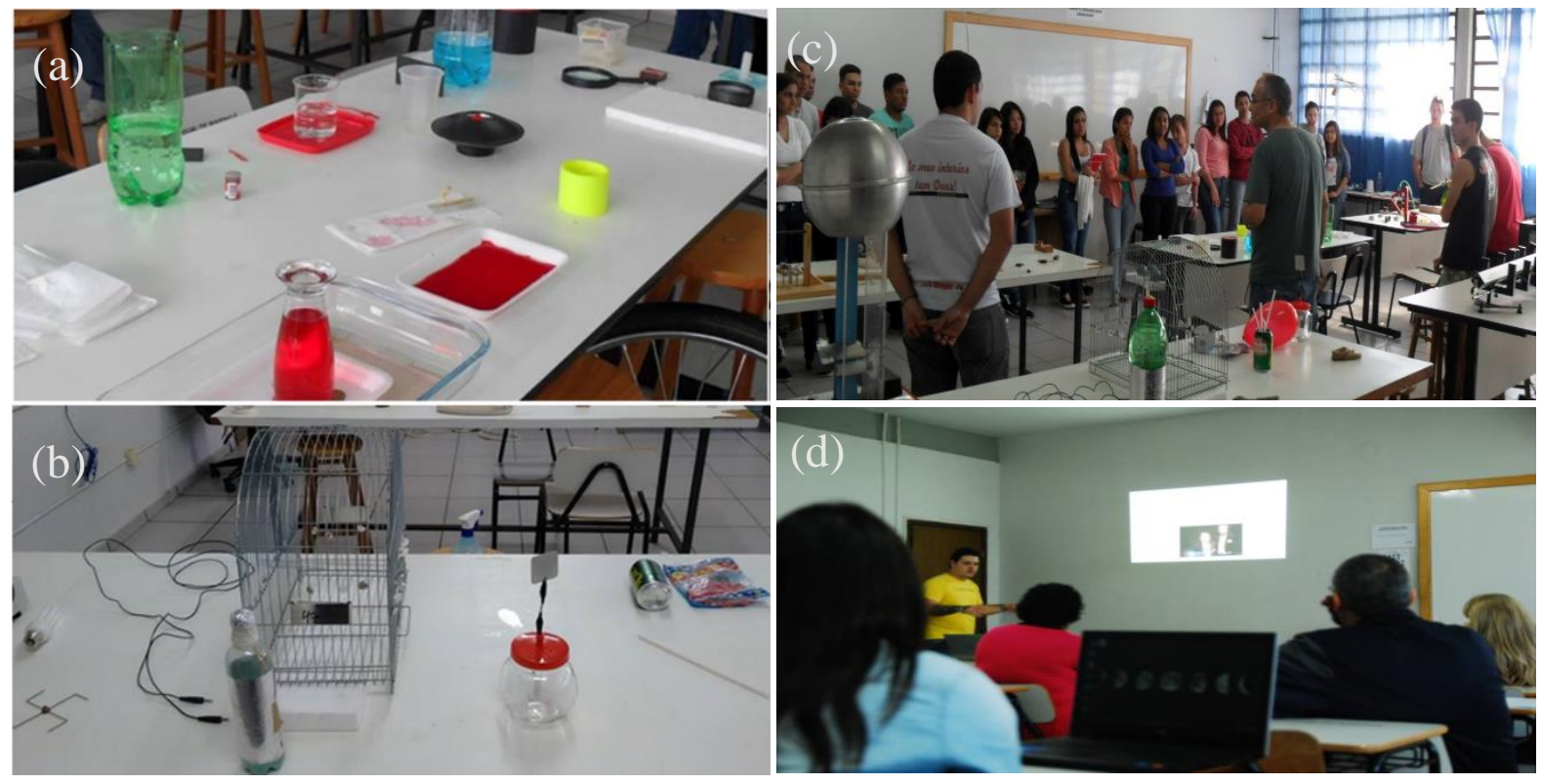

Figura 1. (a) Bancada de experimentos confeccionados com materiais de baixo custo sobre pressão, densidade e ótica; em (b) sobre eletricidade, como gaiola de Faraday, eletroscópio, gerador de Van der Graff, além de algumas lâmpadas e brinquedos; em (c) Exemplo de como se inicia as demonstrações para as visitas e em (d) exemplo de uma apresentação de seminários temáticos realizadas ao grupo.

\footnotetext{
${ }^{1}$ Recentemente um guia foi lançado relacionando os museus de ciência. Centros de Museus de ciência do Brasil em 2015 foi publicado pela Associação Brasileira de Centros e Museus de Ciência (ABCMC), além de outras parcerias. (PIERRO, B. 2015)
} 
Projeto escolas na Universidade Estadual de Maringá: criação de um ambiente de experimentação e exploração em ciências

Posteriormente os graduandos demonstram os experimentos aos alunos e professores visitantes. A figura 2 mostra estudantes frente a algumas demonstrações de mecânica (máquinas simples e energia mecânica) e, como na proposta de um museu dinâmico, livres no ambiente para interação com os experimentos.

Espaço como este oferece aos professores e estudantes do município de Goioerê-PR e região, tanto da rede pública como as instituições particulares de educação básica, mais uma forma de ampliação de suas capacidades criativas e de ação, proporcionando-lhes um ambiente de experimentação e exploração de conceitos científicos, em particular de fenômenos físicos (CEZALLI, 2002).
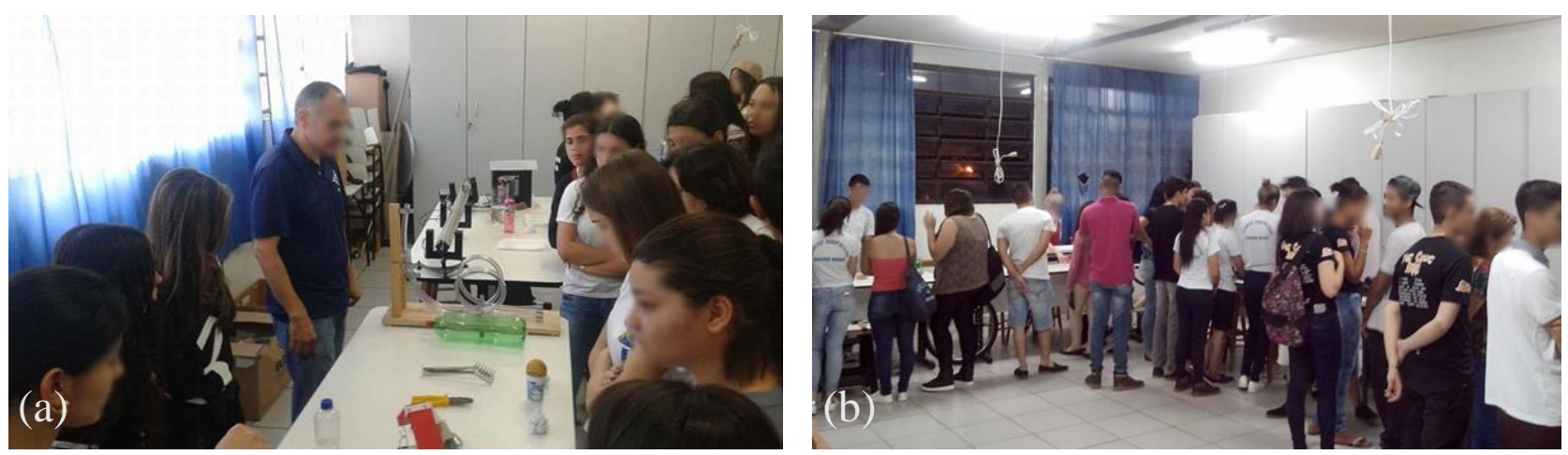

Figura 2. Demonstrações de experimentos de mecânica para alunos do terceiro ano do ensino médio, durante uma visita ao campus da UEM-CRG; em (b) os mesmos alunos interagindo com os experimentos.

Projetos desta natureza permitem que os alunos se aproximem do universo da ciência, desvendando e conhecendo melhor os fenômenos da natureza. E ainda, provocam a curiosidade e o interesse pelo conhecimento. A Figura 3 mostra o fechamento de uma visita de uma escola local ao campus e uma noite de observação celeste.
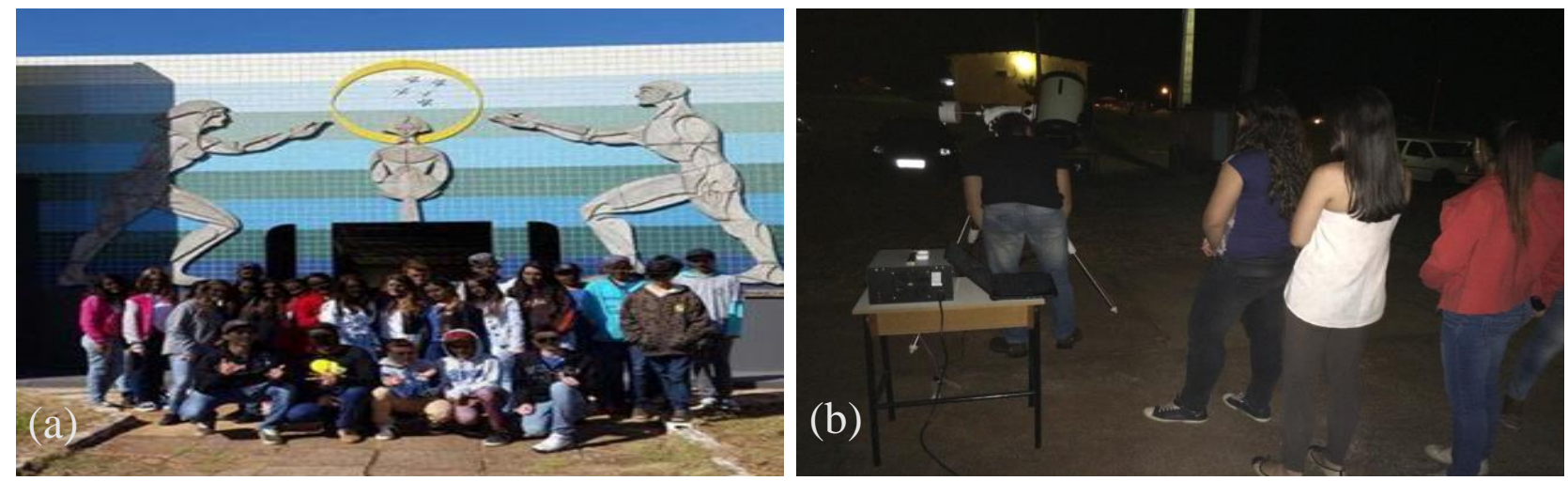

Figura 3. (a)Fechamento de uma visita com alunos do primei ano do ensino médio e em (b) noite de observação realizada durante a participação na feira de profissões do campus. 
Projeto escolas na Universidade Estadual de Maringá: criação de um ambiente de experimentação e exploração em ciências

Visando compreender como o projeto alcança os alunos participantes, levando em consideração o grande número de alunos que visitam o ambiente de demonstrações e por entendemos que projetos desta natureza se descaracterizam de uma pesquisa de ensino, um questionário simples foi aplicado em algumas das visitas (há pouca disponibilidade de tempo), com o objetivo de descobrir como o projeto tem sido aceito pela comunidade. (ALLPORT, $\mathrm{s} / \mathrm{d}$.).

\section{RESULTADOS E DISCUSSÕES.}

Como citado, as questões buscaram compreender como o projeto tem sido aceito. Quando perguntados "o que você achou da visita" encontramos que aproximadamente $70 \%$ (96 alunos) classificaram a atividade como ótima, 20,43\% (28 alunos), como boa, e 9,5\% (13 alunos), como regular. Evidentemente por não termos atingindo todos os alunos, há uma motivação da equipe para ir à busca de novas atividades, com a intenção de alcançar a satisfação para o maior número de alunos possível.

Uma segunda questão, abordando "se os experimentos demonstrados foram interessantes", prospectou índices semelhantes. A maioria, 93\% (128 alunos), afirmou que "sim”, e 7\% (9 alunos) afirmou que "não". Entende-se que este é um bom resultado, comprovando uma boa aceitação do projeto. Nota-se que as questões aplicadas aos alunos oferecem uma maneira de quantificar e interpretar o nível de aceitação das atividades do projeto.

Até o presente momento foram atendidos aproximadamente 300 alunos, entre turmas de ensino fundamental e médio, sendo realizadas diversas demonstrações dos mais variados temas de física.

Com o apoio dos docentes participantes do projeto (professores orientadores), os graduandos do campus ainda incentivam os alunos do ensino fundamental e médio a organizarem feiras de ciências em suas escolas, onde novos experimentos e explicações podem ser produzidos, além de algumas das demonstrações já realizadas neste projeto.

No projeto ESCOLAS NA UEM-CRG, executam-se também atividades como palestras e sessões de cinema, visando melhorias na educação nas escolas locais e da região e a divulgação do ensino de ciências, em particular a Física e a Astronomia. É indispensável um bom entendimento da física e outras ciências para o desenvolvimento de temáticas como, por exemplo, as inovações tecnológicas e suas implicações na evolução da na sociedade, além e muitos outros temas.

Entende-se ainda que nada se compara, no aprendizado de ciências, do que poder observar sua ação e consequência. ou se quer matematizar, sem a necessidade de altos custos para 
Projeto escolas na Universidade Estadual de Maringá: criação de um ambiente de experimentação e exploração em ciências

uma série de efetivas mediações e demonstrações. (GLEISER, 2000; ANGOTTI e AUTH, 2001).

\section{CONSIDERAÇÕES}

Entende-se que a aprendizagem científica deve ser vista como uma construção de conhecimento no decorrer de toda uma vida, em todas as suas etapas. Porém, tem-se observado uma grande lacuna na pesquisa e projetos que abordem a aprendizagem de ciência em locais diferentes do cotidiano escolar. Em nossa atual realidade de ensino, poucas são as oportunidades que os alunos têm de abrigar-se em um ambiente de descobertas e realizar experimentações (RODRIGUES 2009).

Observa-se que a inclusão da experimentação em sala de aula ocorre em poucas instituições de ensino. (GASPAR, 2003 apud MENDONÇA e DIAS, 2016). Com o avanço tecnológico, rapidez e a disseminação das informações, é evidente que a sociedade sofra modificações em diversos aspectos. Com a aprendizagem não deve ser diferente. (MARCELO, 2001; HARGREAVES, 2004). Todavia, um olhar detalhado para dificuldades do ensino de física não faz parte do escopo deste trabalho. Entende-se que elas ocorram continuadamente. Já sabemos que ensinar física não é uma tarefa fácil, aprender menos ainda. (GLEISER, 2000).

O espaço criado pelo projeto, uma vez que nossa região carece de lugares como tal, aproxima o conhecimento científico da sociedade em geral, e contribui para aumentar os debates sobre ciência. Questões como "o que é ciência", "como se faz ciência" e "como está a ciência em nosso país", entre outros, são importantes para a compreensão e a evolução do conhecimento científico dos indivíduos tem sido incentivadas dentro do projeto ESCOLAS NA UEM.

A execução de atividades em ambientes não formais de aprendizagem, que desenvolvam e reconheçam as habilidades cognitivas, estão também correlacionadas à base sociocultural dos estudantes (FLACH \& ANTONELLO, 2010). Neste trabalho foi evidenciada a importância da utilização de demonstrações e do uso de ambientes não formais de aprendizagem, que têm contribuído para melhorar a formação dos estudantes de escolas de ensino médio e fundamental.

Projetos que utilizam a experimentação como aditivo para o processo de ensinoaprendizagem proporcionam aos seus integrantes e espectadores a oportunidade de aproximar a Universidade da comunidade escolar, possibilitando uma melhor integração entre o que é trabalhado nas instituições de ensino superior e o que é trabalhado nas instituições de educação básica. 
Projeto escolas na Universidade Estadual de Maringá: criação de um ambiente de experimentação e exploração em ciências

\section{REFERÊNCIAS}

ALLPORT, G.W. Coleta de dados: II questionários e entrevistas. S/d.

ANGOTTI, J. A. P.; AUTH, M.A. Ciência e tecnologia: implicações sociais e o papel da educação. Ciência \& Educação, v.7, n.1, p.15-27, 2001.

BORGES, A. T. Os novos rumos para o laboratório escolar de ciências. Caderno Brasileiro de Ensino de Física., v. 19, n.3: p.291-313. 2002.

CEZALLI, S. et al. Tendências pedagógicas das exposições de um museu de ciências.In: GUIMARÃES, V.; SILVA, G. A. (Coord.). Implantação do Centro e Museus de Ciências. Rio de Janeiro: UFRJ, 2002. p. 208-218.

CONLON, T. J. A review of informal learning literature, theory and implications for practice in developing global professional competence. Journal of European Industrial Training, Bingley, v. 28, n. 2-4, p. 283-295, 2004.

FLACH, L., ANTONELLO, C. S. A teoria sobre aprendizagem informal e suas implicações nas organizações. Revista Eletrônica de Gestão Organizacional, Recife, v. 8, n. 2, p. 193-208, 2010.

GASPAR, A. Experiências em ciências para o ensino fundamental. São Paulo: Editora Livraria da Física, 2003.

GOHN, M. G., Educação Não-Formal e Cultura Política. Cortez, São Paulo,1999.

HARGREAVES, A. Para além da padronização: comunidades de aprendizagem profissional ou seitas de treinamento para o desempenho? In: O ensino na sociedade do conhecimento: educação na era da insegurança. Trad. COSTA, R. C. Porto Alegre: Artmed, 2004, p. 171-200.

LANGHI, RODOLFO \& NARDI ROBERTO. Ensino de Astronomia no Brasil: Educação formal, informal, não formal e divulgação cientifica. Revista Brasileira de Ensino de Física, v. 31, n. 4, 4402. 2009.

LIVINGSTONE, D.W., Exploring the icebergs of Adult: Findings of the First Canadian. Survey of Informal Learning Practices, Universidade de Toronto (CA), 1999. 
Projeto escolas na Universidade Estadual de Maringá: criação de um ambiente de experimentação e exploração em ciências

MARCELO, C. Aprender a enseñar para la Sociedad del Conocimiento. Revista Complutense de Educación, V. 12, N. 2 p. 531-593. 2001.

MENDONÇA A.S, DIAS G.C. O CENTRO DE CIÊNCIAS: uma ferramenta para aprendizagem científica informal na prática docente. São Paulo. Blucher Open Acess. 2016.

MARANDINO, M.; SILVEIRA, R. V. M.; CHELINI, M. J.; FERNANDES, A. B.; RACHID, V.; MARTINS, L. C.; LOURENÇO, M. F.; FERNANDES, J. A.; FLORENTINO, H. A. A educação não-formal e a divulgação científica - o que pensa quem faz. In: Encontro Nacional de Pesquisa em Educação em Ciências, 4, 2003, Bauru, Atas, Bauru, 2003

MARDEGAN, F. Aprendizagem informal: como os indivíduos aprendem em seus locais de trabalho? Disponível em: http://www.abd.org.br/abd/f01/docs/artigos/2013/260313/aprendizagem-informal.pdf. Acesso em 23 de março de 2016.

MOORE, T. W. Philosophy of Education: An Introduction. Londres, pp. 74-76. 1982.

PIERRO B. Ciência sobre Rodas: Guia mapeia 32 museus científicos itinerantes no Brasil Revista Fapesp: Política C\&T Difusão Ed. 234. P30-33. (agosto) 2015

RODRIGUES, D.C.G. A. A Inserção de Atividades Experimentais no Ensino de Ciências em Nível Médio: Um Relato de Sala de Aula. Revista PRAXIS: v1, n.2. 2009

SANTOS, J. H. M., PEREIRA, F. N.V., PENIDO, M. C. M. (2011). Proposta de sequência didática para o Ensino de Astronomia no Fundamental; conhecendo a Lua. Disponível em <http://www.nutes.ufrj.br/abrapec/viiienpec/>Acesso em 02 de janeiro de 2016. 